\title{
Surface Plasmon Singularities
}

\section{Gabriel Martínez-Niconoff, ${ }^{1}$ P. Martinez-Vara, ${ }^{2}$ G. Diaz-Gonzalez, ${ }^{1}$ J. Silva-Barranco, ${ }^{1}$ and A. Carbajal-Domínguez ${ }^{3}$}

\author{
${ }^{1}$ Departamento de Óptica, Instituto Nacional de Astrofísica Óptica y Electrónica (INAOE), Apdo. Postal 51 y 216, \\ Tonantzintla, PUE, Mexico \\ ${ }^{2}$ Facultad de Ingeniería, Benemérita Universidad Autónoma de Puebla (BUAP), Publa, PUE, Mexico \\ ${ }^{3}$ Universidad Juárez Autónoma de Tabasco, (UJAT), Cunduacán, Tab, Mexico
}

Correspondence should be addressed to Gabriel Martínez-Niconoff, gmartin@inaoep.mx

Received 5 April 2012; Revised 12 June 2012; Accepted 13 June 2012

Academic Editor: Zhaolin Lu

Copyright (C 2012 Gabriel Martínez-Niconoff et al. This is an open access article distributed under the Creative Commons Attribution License, which permits unrestricted use, distribution, and reproduction in any medium, provided the original work is properly cited.

\begin{abstract}
With the purpose to compare the physical features of the electromagnetic field, we describe the synthesis of optical singularities propagating in the free space and on a metal surface. In both cases the electromagnetic field has a slit-shaped curve as a boundary condition, and the singularities correspond to a shock wave that is a consequence of the curvature of the slit curve. As prototypes, we generate singularities that correspond to fold and cusped regions. We show that singularities in free space may generate bifurcation effects while plasmon fields do not generate these kinds of effects. Experimental results for free-space propagation are presented and for surface plasmon fields, computer simulations are shown.
\end{abstract}

\section{Introduction}

The contemporary trends in plasmon optics consist of establishing a parallelism with the traditional optical models. The wave nature of the electromagnetic field in both cases implies that the mathematical analysis may present many similarities. However, the physical features may be completely different. An important common behavior is that the electromagnetic field is organized around the singular regions. The simplest singular region is kind fold, and the union of twofold focusing regions generates a cusped focusing region [1]. These are the only focusing regions that can be detected on a plane $[1,2]$, so that they are the expected singularities for surface plasmon fields. From this fact, some physical features can be analyzed. For optical fields in free space, the inverse process may occur, that is, when a cusped focusing region is split into two folds, vortex and bifurcation effects may occur [3]. For the case of plasmon fields, these features do not appear; however, the focusing region generates charge redistribution $[4,5]$. This latter property offers many interesting applications; for example, it can be implemented to polarize particles, allowing the design of plasmonic tweezers.

In the present study, we describe the synthesis of singular regions propagating in free space and they are compared with the singular regions for surface plasmon fields. In both cases, the singularities geometry can be obtained from the phase function $[6,7]$, and it satisfies the nonlinear partial differential equation [6]

$$
\frac{\partial^{2} L}{\partial x^{2}} \frac{\partial^{2} L}{\partial y^{2}}-\left(\frac{\partial^{2} L}{\partial x \partial y}\right)^{2}=0
$$

where $L$ is the optical path length. When the boundary condition is a slit-shaped curve, (1) has a simple geometrical interpretation. The singularity is generated by means of the envelope region of a ray set, and each ray must satisfy the Fermat principle [8], and this construction is sketched in Figure 1. According to the transversal condition [9], the rays must emerge perpendicular to the curve slit. In the case of plasmon fields, this geometrical point of view is very important because all the electromagnetic fields propagating 


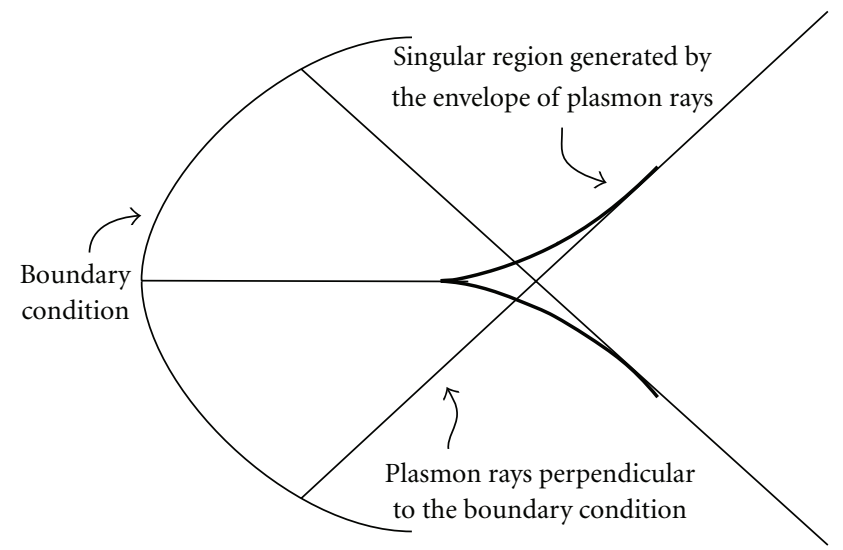

Figure 1: Generation of the singular region by means of a set of plasmon rays. Each ray must satisfy variational principles; this means that the rays must emerge perpendicular to the boundary condition. This construction is valid for waves in free space and surface plasmon fields.

on the metal surface can be considered as a "Geodesic Flow" [10], and in this sense, the singularities are deeply related to the stability of the flow.

\section{Long-Range Surface Plasmon Modes}

Surface plasmon modes are nonhomogeneous electromagnetic waves propagating on the interface of a dielectric-metal media. For a semi-infinite media, the electric field is

$$
\begin{aligned}
& E_{1}(x, z)=\left(\overrightarrow{i a_{1}}+\vec{k} b_{1}\right) \exp \left(-\alpha_{1} z\right) \exp (i \beta x), \\
& E_{2}(x, z)=\left(\overrightarrow{i a_{2}}+\vec{k} b_{2}\right) \exp \left(-\alpha_{2} z\right) \exp (i \beta x) .
\end{aligned}
$$

Related to this electromagnetic mode is the charge motion of the free electrons, that oscillate longitudinally and transversally to the surface. These oscillations are determined by the trajectory of the electric field, whose geometry can be obtained by establishing an analogy with the classical polarization model. It is easy to show that the trajectory of the electric field on the $x-z$ plane is given by

$$
\frac{E_{x}^{2}}{a_{1}^{2}}+\frac{E_{z}^{2}}{\left(\alpha a_{1} /|\beta|\right)^{2}}-\frac{2 E_{x} E_{z}}{a_{1}^{2} \alpha /|\beta|} \cos \delta_{0}=\exp (-2 \alpha z) \sin ^{2} \delta_{0}
$$

where $\beta$ is the dispersion relation function that satisfies

$$
\beta=\frac{w}{c}\left(\frac{\varepsilon_{1} \varepsilon_{2}}{\varepsilon_{1}+\varepsilon_{2}}\right)^{1 / 2} .
$$

$\varepsilon_{1}$ is the permittivity of the dielectric media, $\varepsilon_{2}$ is the permittivity of the metal, which is a complex number, and consequently, $\beta$ is a complex function. The imaginary part is responsible for surface plasmon modes propagating at very short distances, about $100 \mu \mathrm{m}$. This length of propagation is a serious limitation for the development of plasmon optics. The synthesis of focusing regions implies the generation of long-range surface plasmon modes modifying the dispersion relation function $\beta$. This is possible when the surface plasmon is propagating in a metallic thin film of thickness $20-80 \mathrm{~nm}$. The calculus of the new dispersion relation function is described by means of the interaction between two plasmon modes, as shown in Figure 2.

The interaction between the two modes is performed by using the coupling mode theory. This implies to model the surface effects, associating an expression for the refractive index, in the neighborhood of the interface of the dielectricmetal surface, which is given by

$$
n=n_{1}+a \exp (-p z), \quad z \geq d,
$$

where $n_{1}$ is the refractive index of the dielectric media, $a$ is a small constant determined by the combination of the materials involved, $d$ is the thickness of the film, and $p$ is a parameter that allows us to model the surface effects. When $z \rightarrow \infty$, the proposed refractive index recovers the classical expression for the dielectric media.

For this case, the dispersion relation function acquires the form

$$
\beta=\frac{\omega}{c}\left\{\left(\frac{\varepsilon_{1} \varepsilon_{2}}{\varepsilon_{1}+\varepsilon_{2}}\right)^{1 / 2}+2 a n_{1}\left(\frac{\varepsilon_{1} \varepsilon_{2}}{\varepsilon_{1}+\varepsilon_{2}}\right)^{-1 / 2} \exp (-2 \alpha d)\right\} .
$$

The calculus details can be found in [4]. When the last expression is substituted in (3), the trajectory geometry of the electrical field on $z=$ constant is an ellipse segment, as shown in Figure 3.

The modulus of the imaginary part given by (6) is minor, when compared with that given by (4). As a consequence the plasmon fields for a thin film propagate large distances. The geometrical interpretation is that the $x$-component for the electric field is minor, decreasing the amplitude oscillation of the charge along the $x$-axis.

The parameters implicit in (5) are sketched in Figure 2. The expression for the long-range surface plasmon mode is obtained by replacing the expression for $\beta$ given by (6) in (2).

To generate the singular regions, it is necessary to describe the long-range plasmon mode on the $x-y$ plane. The corresponding expression is obtained by rotating the $z$-axis, and the transformation of coordinates is $z \rightarrow z$; $x \rightarrow x \cos \theta \pm y \sin \theta$. The general expression for the plasmon mode is

$$
\begin{aligned}
E(x, y, z)= & (\vec{i} a+\vec{k} b \cos \theta+\vec{j} b \sin \theta) \\
& \times \exp \left(-\alpha_{1} z\right) \exp (i \beta(x \cos \theta+y \sin \theta)),
\end{aligned}
$$

with $\beta$ given as that presented in (6).

\section{Singular Regions in Free Space}

With the purpose to analyze the singularities, we describe the generation of fold singularities propagating in free space. The transmittance function has a mathematical representation in the frequency space of the form [6]

$$
T(u, v)=\delta(u-f(v)),
$$




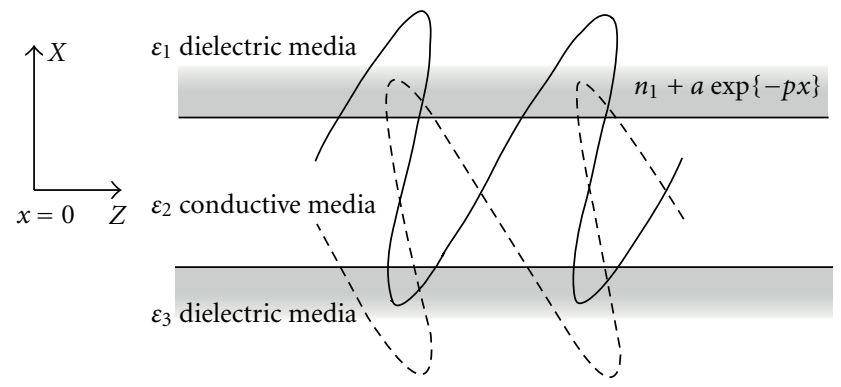

FIGURE 2: Schematic set up to describe the synthesis of long-range surface plasmon modes. The surface effects are described by assuming an exponential representation for the refractive index. The physical effects are described by assuming two surface plasmon modes propagating on each surface.

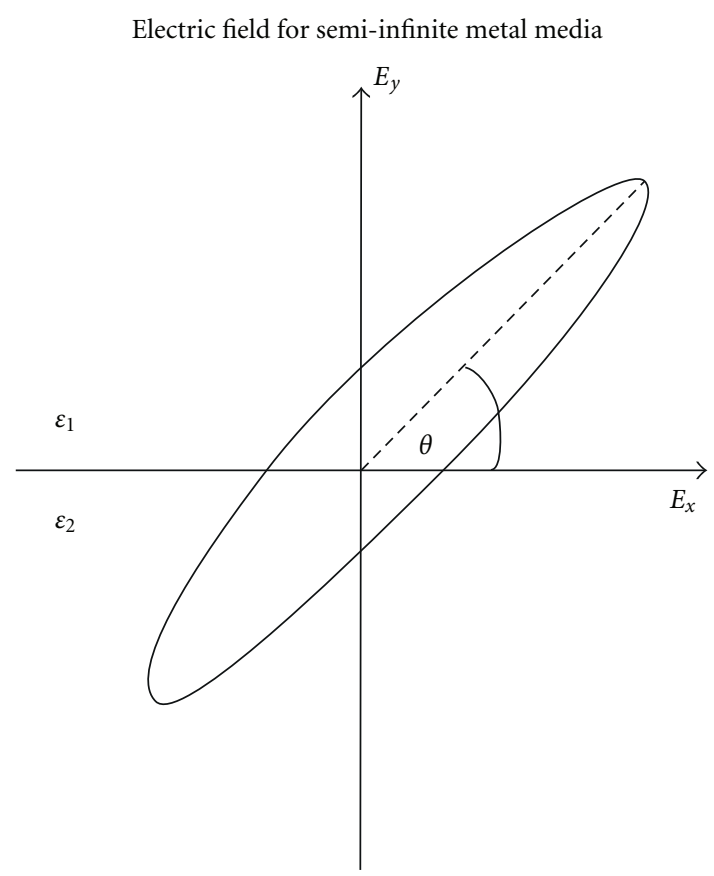

(a)
Electric field for thin metal film

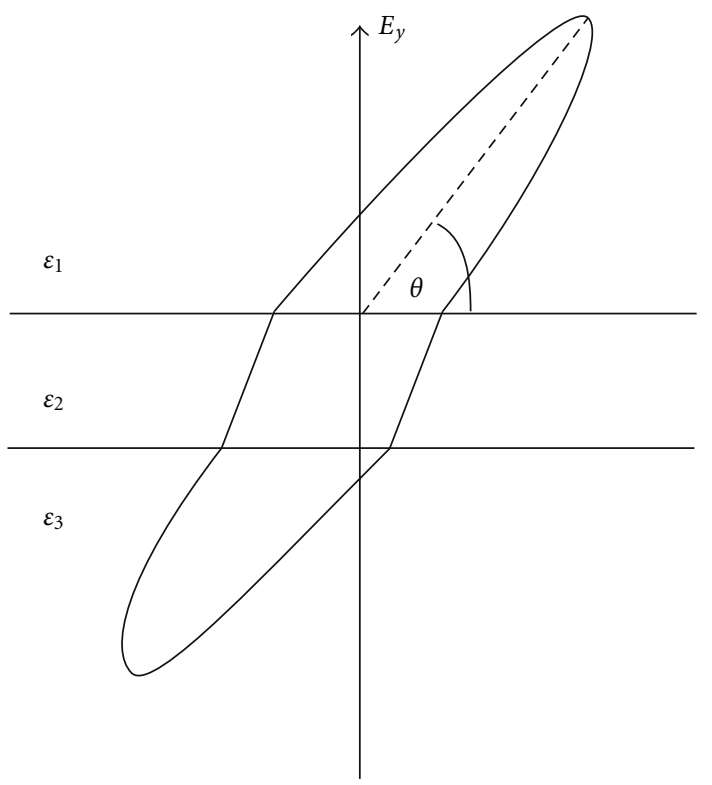

(b)

FIgURE 3: Polarization-like trajectory for surface plasmon modes. (a) Surface plasmon modes propagating in a semi-infinite media. (b) Surface plasmon modes propagating on a thin film.

where $\delta$ is the Dirac delta-function. The diffraction field, using the angular spectrum model is given by [11]

$$
\begin{aligned}
\varphi(x, y, z)=\iint_{-\infty}^{\infty} \delta(u-f(v)) \\
\quad \times \exp \{i 2 \pi(x u+y v+z p)\} d u d v
\end{aligned}
$$

where $u=\cos \alpha / \lambda, v=\cos \beta / \lambda, p=\cos \gamma / \lambda$ are the spatial frequencies that satisfy $u^{2}+v^{2}+p^{2}=1 / \lambda^{2} ; \alpha, \beta$, and $\gamma$ are the angles that makes the wave vector with the $(x, y, z)$-axis; and $\lambda$ is the wavelength. In Figure 4, the setup to generate the diffraction field associated with slit-shaped curves is shown.

As prototype, we describe the synthesis of two kinds of optical fields. The first one has a transmittance as boundary condition, whose geometry in frequency space is a cubic function given by

$$
T(u, v)=\delta\left(u-v^{3}\right) .
$$

The optical field has the representation

$$
\begin{aligned}
& \varphi(x, y, z) \\
& \quad=\iint_{-\infty}^{\infty} \delta\left(u-v^{3}\right) \exp \{i 2 \pi(x u+y v+z p)\} d u d v \\
& \quad=\int_{-\infty}^{\infty} \exp \left\{i 2 \pi\left(x v^{3}+y v+z\left(\frac{1}{\lambda^{2}}-\left(v^{6}+v^{2}\right)\right)^{1 / 2}\right)\right\} d v .
\end{aligned}
$$

The optical field presents bifurcation effects that are manifested during its propagation. To understand the amplitude 


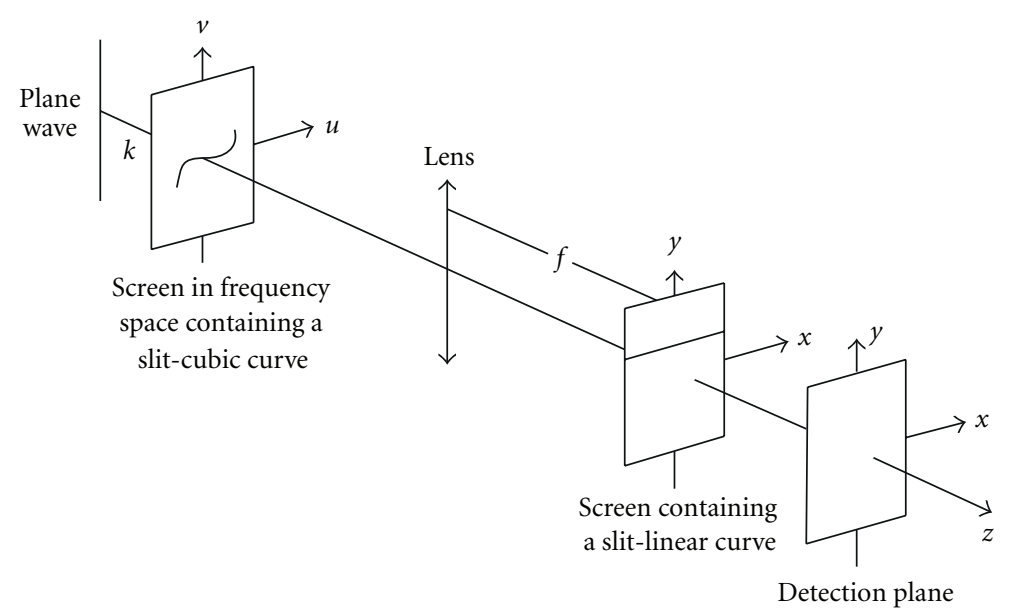

FIGURE 4: Schematic setup to generate the optical field associated with slit-shape curves.

distribution, we implement a kind of spatial filtering, which is obtained by placing a screen containing a linear slit on $z=0$, whose representation is $t(y)=\delta(y-1)$. The amplitude distribution is

$$
\begin{aligned}
& \Phi_{1}(x, y, z=0) \\
& =\delta(y-1) \int_{-\infty}^{\infty} \exp i 2 \pi\left(x v^{3}+y v\right) d v \\
& =\delta(y-1) \int_{-\infty}^{\infty} \exp \left(i 2 \pi\left(x v^{3}+v\right)\right) d v .
\end{aligned}
$$

By translating the screen to the position defined by $y=-1$, the mathematical representation for the optical field is given by

$$
\begin{aligned}
& \Phi_{2}(x, y, z=0) \\
& \quad=\delta(y+1) \int_{-\infty}^{\infty} \exp \left(i 2 \pi\left(x v^{3}-y v\right)\right) d v \\
& =\delta(y+1) \int_{-\infty}^{\infty} \exp \left(i 2 \pi\left(x v^{3}-v\right)\right) d v .
\end{aligned}
$$

The phase function represented by (12) and (13) differs in a sign, being equals along the direction defined by $v=$ 0 . The bifurcations effects appear when the optical field is propagating to different planes, that is, $z \neq 0$; therefore, each optical field propagates by different paths. In Figure 5(a), the experimental result in $z=0$ plane is shown, and in Figure 5(b) the experimental result for $z \neq 0$ where the bifurcation effects become evident are presented. From these results, it can be noted that the right branch is shifted to the bottom and the left branch is shifted to the top. The entire optical field unfolds based on the structure of the transmittance function.

\section{Singular Surface Plasmon Fields}

Singularities for surface plasmon fields can be obtained in a way similar to that employed for the free space case, and the corresponding surface plasmon angular spectrum model is $[4,5]$

$$
\begin{aligned}
& E_{1}(x, y, z) \\
& \quad=\iint_{-\infty}^{\infty}(\vec{i} A(u)+\vec{j} B(u)+\vec{k} C(u)) \\
& \quad \times \exp \left(-2 \pi \alpha_{1} z\right) \exp (i 2 \pi \beta(x u+y v)) d u d v,
\end{aligned}
$$

where the term $(\vec{i} A(u)+\vec{j} B(u)+\vec{k} C(u))$ represents the transmittance function in the frequency space. For a slitshape curve, we have that the transmittance takes the form

$$
T(u, v)=(\vec{i} a+\vec{j} b+\vec{k} c) \delta(u \tau(\lambda, d)-f(v)),
$$

where $(a, b, c)$ are the components of a constant vector and are necessary to satisfy the boundary conditions of the electromagnetic field. More details with respect to the value of the constants can be found in [4]. The scale term $\tau(\lambda, d)$ is proposed to describe the coupling between the diffraction field propagating in free space and surface plasmon fields. In Figure 6, the experimental setup to generate surface plasmon singularities is shown.

The singularities are generated by illuminating a slitshape curve recorded on a thin metal film, whose thickness is in the order of $20-80 \mathrm{~nm}$, and this allows generating longrange surface plasmon modes. The width $d$ of the slit must be smaller than the illumination wavelength $\lambda$.

The expression for the surface plasmon diffraction field is given by

$$
\begin{aligned}
E_{1}(x, y, z)=\int_{-\infty}^{\infty}(\vec{i} a+\vec{j} b+\vec{k} c) \exp (-2 \pi \alpha z) \\
\quad \times \exp (i 2 \pi \beta(x \tau(\lambda, d) f(v)+y v)) d u .
\end{aligned}
$$

Let us consider two examples. The first one is when the transmittance function contains a slit-cubic curve, given by

$$
T(u, v)=(\overrightarrow{i a}+\vec{j} b+\vec{k} c) \delta\left(u\left(\frac{\lambda}{d}+i \frac{d}{\lambda}\right)-v^{3}\right),
$$




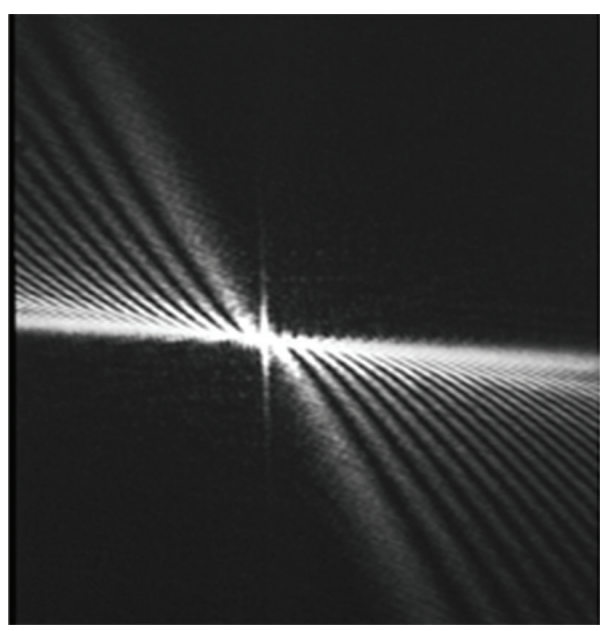

(a)

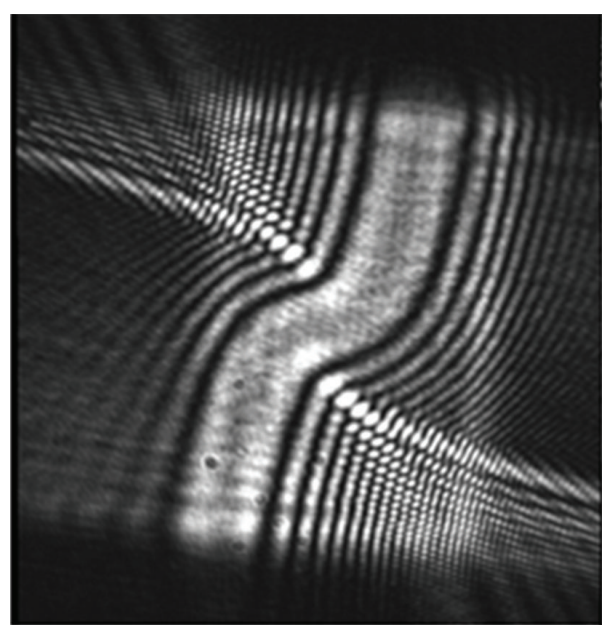

(b)

Figure 5: (a) Optical field on $z=0$ plane. This optical field is formed by two optical fields, and the spatial evolution of these two fields generates the bifurcation effects. (b) The generation of bifurcation effects. The distance between the two images along $z$-coordinate is approximately $1 \mathrm{~cm}$; after this distance, the optical field remains practically unchanged.

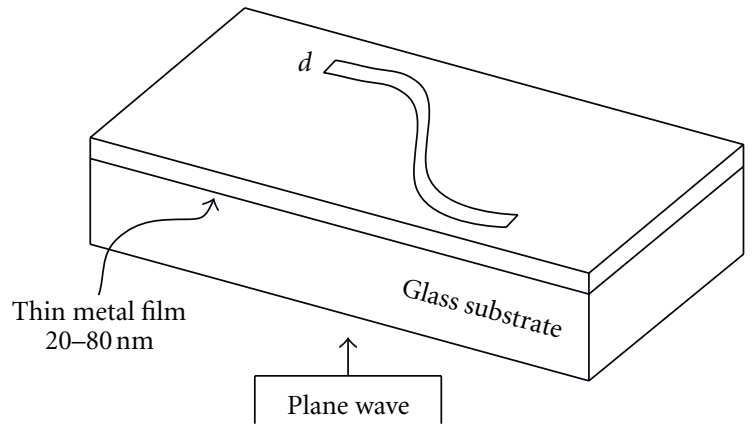

Figure 6: Experimental setup to generate surface plasmon singularities. The thin metal film contains a slit-shaped curve.

The term $\tau(\lambda, d)=(d / \lambda+i(\lambda / d))$ is proposed as an ansatz. When $d>>\lambda$, the imaginary part is very small and the optical field recovers the structure of the optical field propagating in free space. The opposite case $d<<\lambda$, means that the imaginary part becomes dominant and the optical field acquires the form of evanescent waves, wich is the condition to generate surface plasmon fields. The surface plasmon diffraction field is

$$
\begin{aligned}
E_{1}(x, y, z)= & \int_{-\infty}^{\infty}(\vec{i} a+\vec{j} b+\vec{k} c) \exp (-2 \pi z \alpha) \\
& \times \exp \left(i 2 \pi \beta\left(y \tau(\lambda, d) v+x \tau(\lambda, d v)^{3} f(v)^{3}\right)\right) d v
\end{aligned}
$$

having a representation of a fold caustic [2] and is similar to an Airy function [12]. The second example is when the transmittance function contains a slit-quadratic curve given by

$$
T(u, v)=(\overrightarrow{i a}+\vec{j} b+\vec{k} c) \delta\left(v \tau(\lambda, d)-u^{2}\right)
$$

and the surface plasmon diffraction field is

$$
\begin{aligned}
& E_{1}(x, y, z) \\
& \quad=\int_{-\infty}^{\infty}(\overrightarrow{i a}+\overrightarrow{j b}+\vec{k} c) \delta\left(v \tau(\lambda, d)-u^{2}\right) \\
& \quad \times \exp (-2 \pi \alpha z) \exp (i 2 \pi \beta(y v+x u)) d u .
\end{aligned}
$$

The amplitude distribution must satisfy the Helmholtz equation, which means that the spatial frequencies must satisfy $\beta^{2}\left(u^{2}+v^{2}\right)-\alpha^{2}=1 / \lambda^{2}$. By substituting this expression in the integral representation and using the paraxial approximation, the amplitude function acquires the form

$$
\begin{aligned}
& E_{1}(x, y, z) \\
& =\int_{-\infty}^{\infty}(\overrightarrow{i a}+\overrightarrow{j b}+\vec{k} c) \delta\left(v \tau(\lambda, d)-u^{2}\right) \exp (-2 \pi \alpha z) \\
& \quad \times \exp \left(i 2 \pi \beta\left[y\left(\frac{1}{\lambda \beta}+\frac{\lambda \beta}{2}\left(\alpha^{2}-u^{2}\right)\right)+x u\right]\right) d u \\
& E_{1}(x, y, z) \\
& =\int_{-\infty}^{\infty}(\overrightarrow{i a}+\overrightarrow{j b}+\overrightarrow{k c}) \exp (-2 \pi \alpha z) \\
& \quad \times \exp \left(i 2 \pi \beta\left[y\left(\frac{1}{\lambda \beta}+\frac{\lambda \beta}{2}\left(\alpha^{2}-\frac{u^{4}}{\tau^{2}}\right)\right)+x \frac{u^{2}}{\tau}\right]\right) d u .
\end{aligned}
$$

In Figure 7, we show the computer simulation. At this point, it is convenient to compare (9) with (16). In both cases, the boundary conditions are described in the $x-y$ plane. However the phase terms in each case are different, having a decreasing exponential function for plasmon fields and a complex dependence for free-space propagation. This means 


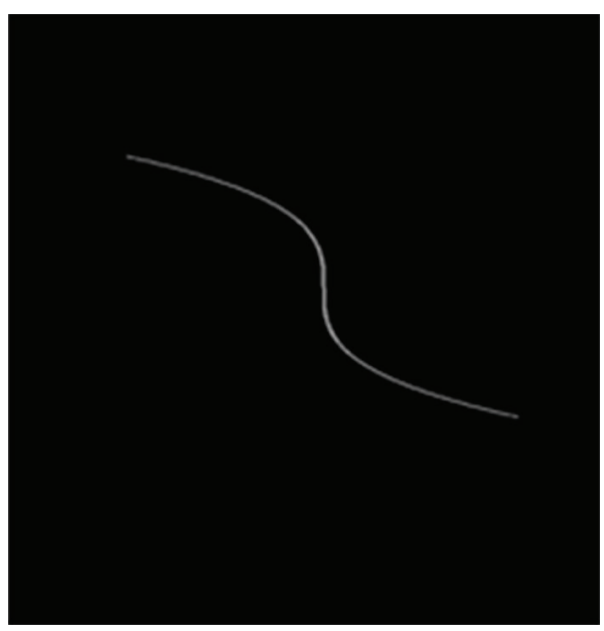

(a)

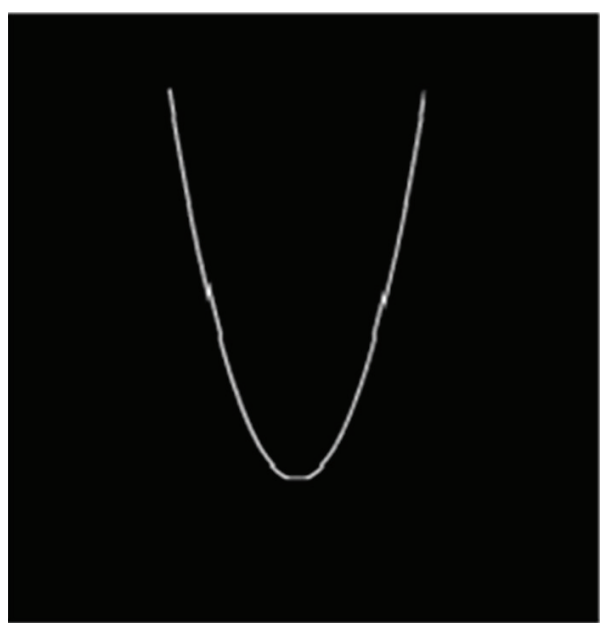

(c)

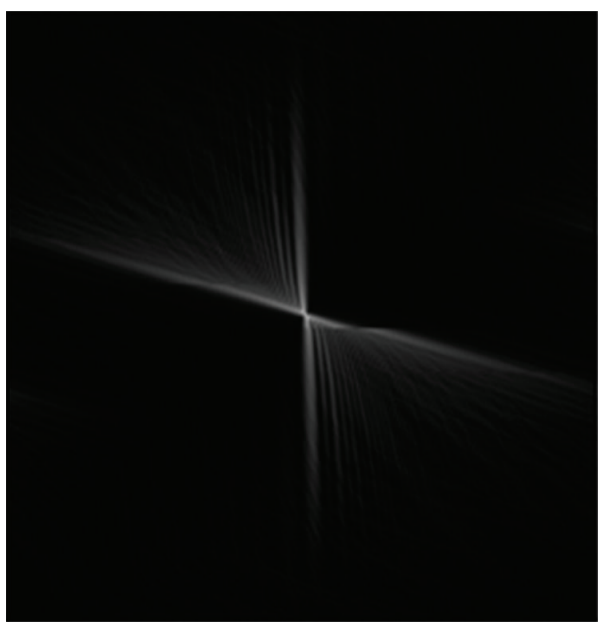

(b)

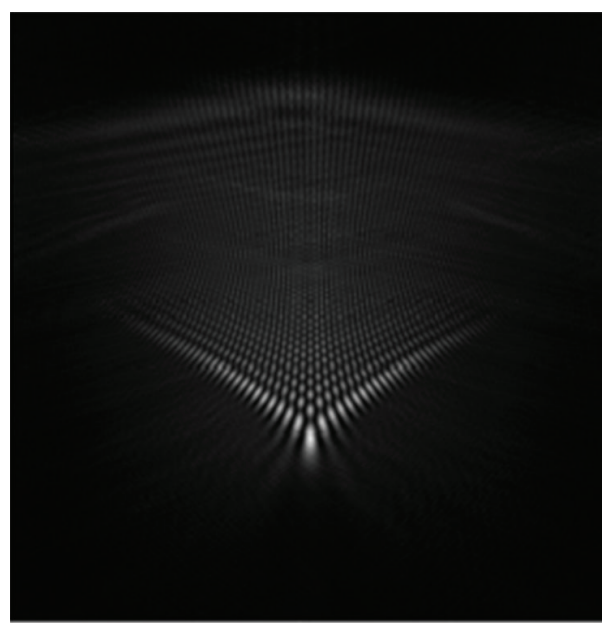

(d)

FIgURE 7: Front view of the metal surface. The illumination is incident from the back of the screen. (a) The screen containing a slit-cubic curve recorded on a thin metal film. (b) The surface plasmon diffraction field and its singular region. (c) Transmittance containing a parabolic curve and (d) singularity associated with this transmittance.

that the bifurcation effects must appear perpendicular to the plane that contains the slit-shaped curve; consequently, the plasmon singularities have a stationary character.

Finally, we remark that the phase function in (18) and (21) has the structure of a catastrophe function [2]. This means that the singularities correspond to the curvature center of the slit curve [6], and has a stationary character, generating charge redistribution given by $\nabla \cdot E_{1,2}=\rho / \varepsilon_{1,2}$, where the electrical field can be represented as

$$
\begin{aligned}
& E(x, y, z) \\
& =\int_{-\infty}^{\infty} \vec{\xi} \exp (-2 \pi \alpha z) \\
& \quad \times \exp (i 2 \pi \beta[\text { germ function }(v) \\
& \quad+\text { perturbation function }(\nu, m, n)] d v),
\end{aligned}
$$

where $m, n$ are parameters related with the curvature centers. The generic physical properties associated to the singularities of surface plasmon fields can be obtained from this representation.

\section{Conclusions}

We described the synthesis of surface plasmon singularities by analyzing the spatial evolution of the surface plasmon field emerging from a slit-shaped curve. The singularities can be easily understood by considering that they are generated by an envelope of a set of normal trajectories emerging from each point on the boundary condition. The geometry generated by the envelope of trajectories satisfies a nonlinear partial differential equation, and the surface plasmon singularity generates a charge distribution; for this reason, the singularities offer application as surface plasmon tweezers. This can be implemented by depositing micronano/particles 
on the metal surface, illuminating with different frequencies; we can generate a set of plasmon singularities inducing tunable resonance effects. These results and its potential applications will be presented in a forthcoming paper.

\section{References}

[1] V. I. Arnold, Singularities of Caustics and Wave Fronts, Kluwer Academic, 1990.

[2] M. V. Berry and C. Upstill, "Catastrophe optics: morphologies of caustics and their diffraction patterns," in Progress in Optics, E. Wolf, Ed., vol. 18, North-Holland, 1980.

[3] Y. S. Kivshar and E. A. Ostrovskaya, "Optical vortices: folding and twisting waves of light," Optics and Photonics News, vol. 12, no. 4, pp. 24-29, 2001.

[4] G. Martínez Niconoff, P. Martínez Vara, J. Munoz-Lopez, J. C. Juárez-Morales, and A. Carbajal-Dominguez, "Partially coherent surface plasmon modes," Journal of the European Optical Society, vol. 6, p. 52, 2011.

[5] G. Martinez Niconoff, J. A. Sanchez-Gil, H. H. Sanchez, and A. P. Leija, "Self-imaging and caustics in two-dimensional surface plasmon optics," Optics Communications, vol. 281, no. 8, pp. 2316-2320, 2008.

[6] G. Martinez-Niconoff, J. Carranza, and A. CornejoRodriguez, "Caustics of diffraction fields," Optics Communications, vol. 114, no. 3-4, pp. 194-198, 1995.

[7] G. Martínez-Niconoff, J. Muñoz-Lopez, and E. MéndezMartínez, "Description of phase singularities and their application to focusing design," Journal of the Optical Society of America A, vol. 18, no. 9, pp. 2089-2094, 2001.

[8] M. S. Sosskin and M. V. Vasnetsov, "Singular Optics," in Progress in Optics, E. Wolf, Ed., vol. 42, North-Holland, 2001.

[9] L. Elgoltz, Differential Equations and Variational Calculus, Mir, Moscow, Russia, 1977.

[10] F. Papoff and R. Zambrini, "Convective instability induced by nonlocality in nonlinear diffusive systems," Physical Review Letters, vol. 94, no. 24, Article ID 243903, 4 pages, 2005.

[11] J. P. Goodman, Introduction to Fourier Optics, McGraw-Hill, 2000.

[12] W. Liu, D. N. Neshev, I. V. Shadrivov, A. E. Miroshnichenko, and Y. S. Kivshar, "Plasmonic Airy beam manipulation in linear optical potentials," Optics Letters, vol. 36, no. 7, pp. 1164-1166, 2011. 

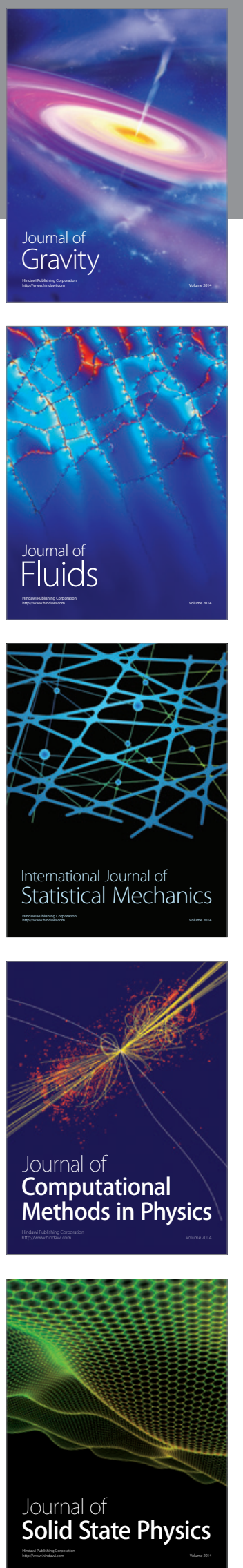

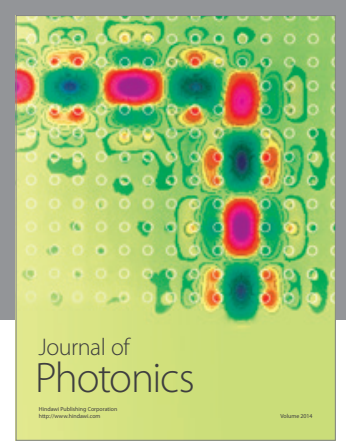

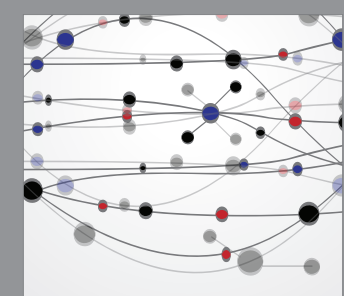

The Scientific World Journal
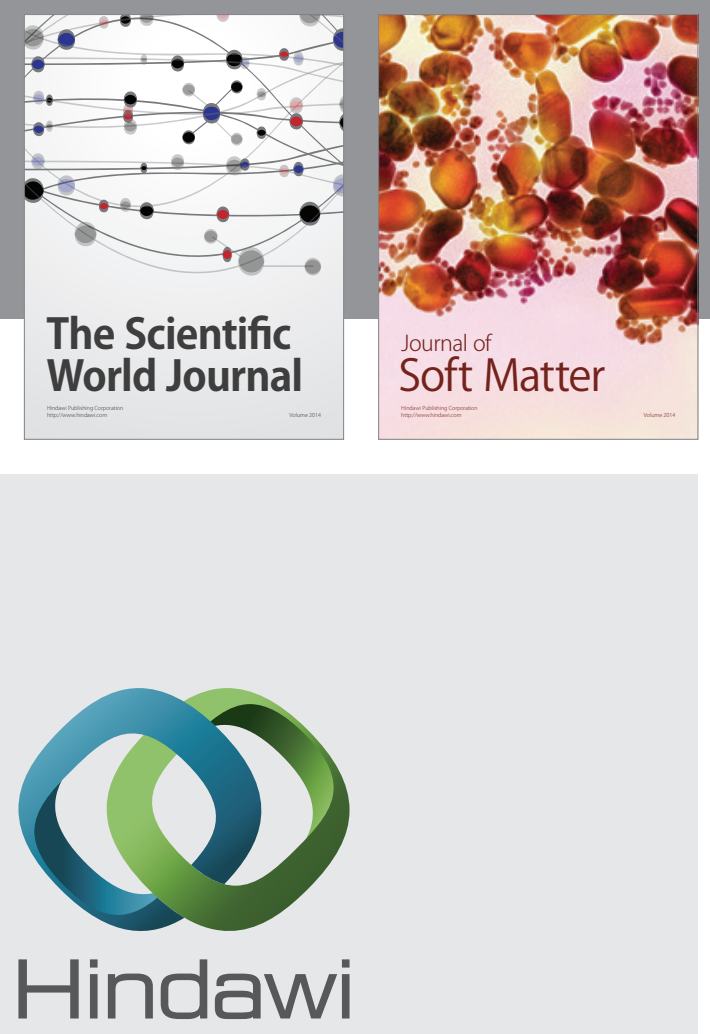

Submit your manuscripts at

http://www.hindawi.com
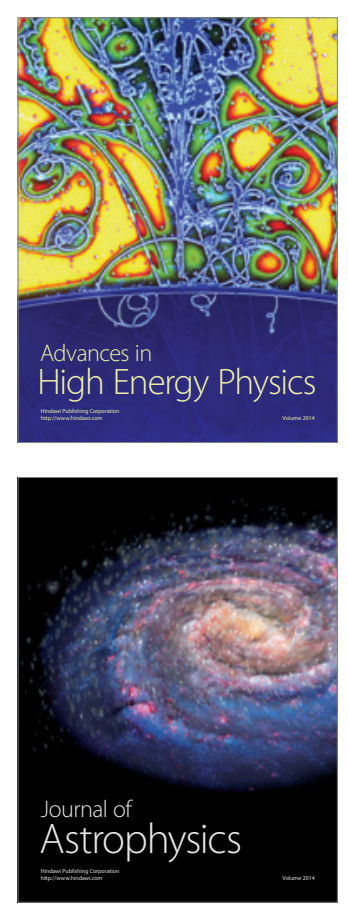
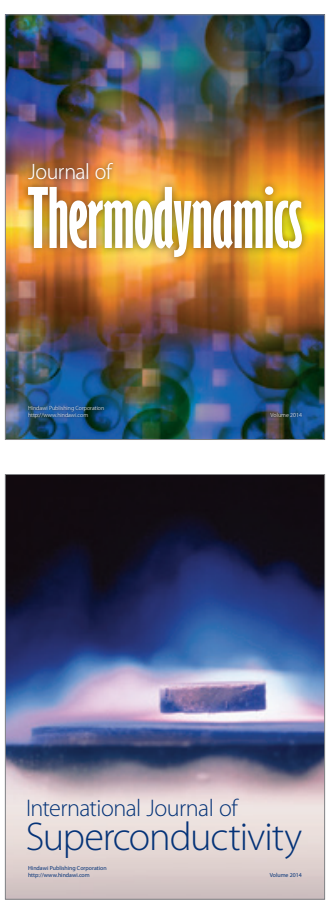
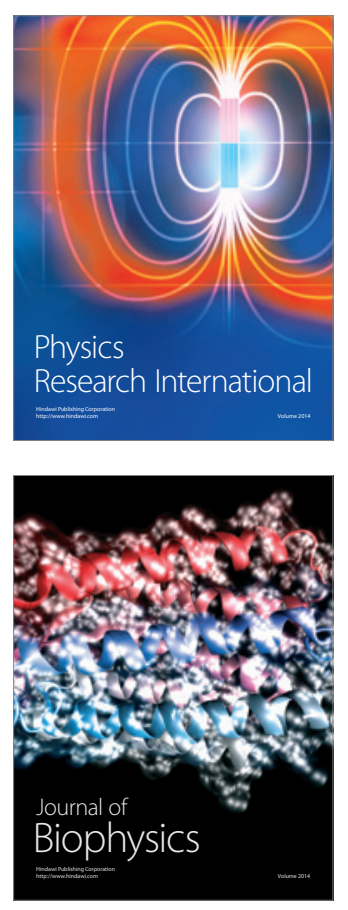
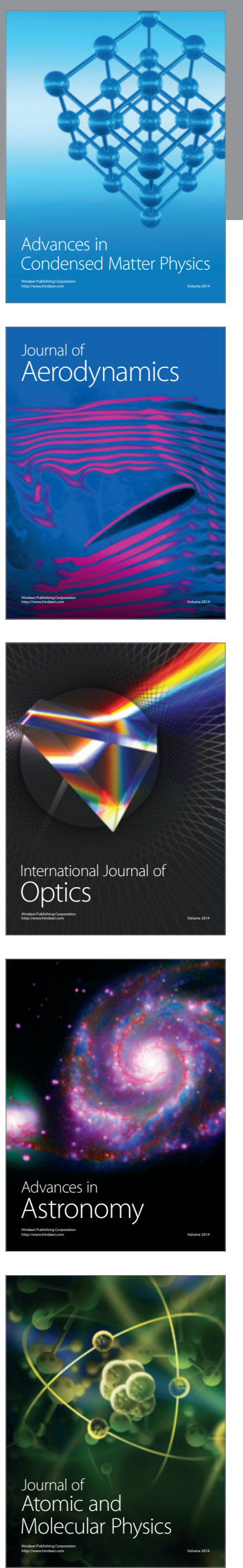